\title{
Adaptive and maladaptive expression plasticity underlying herbicide resistance in an agricultural weed.
}

Emily B. Josephs ${ }^{1,2 *}$, Megan L. Van Etten ${ }^{3,4}$, Alex Harkess ${ }^{5,6}$, Adrian Platts ${ }^{1}$, Regina S. Baucom ${ }^{3 *}$

1 Department of Plant Biology, Michigan State University, East Lansing, MI

2 Ecology, Evolution, and Behavior Program, Michigan State University, East Lansing, MI

3 Department of Ecology and Evolutionary Biology, University of Michigan, Ann Arbor, MI

4 Biology Department, Pennsylvania State University, Dunmore, PA, 18512

5 Department of Crop, Soil, and Environmental Sciences, Auburn University, Auburn, AL, 36849

6 HudsonAlpha Institute for Biotechnology, Huntsville, AL, 35806

* Corresponding authors

\begin{abstract}
Plastic phenotypic responses to environmental change are common, yet we lack a clear understanding of the fitness consequences of these plastic responses.Here, we use the evolution of herbicide resistancein the common morning glory (Ipomoea purpurea) as a model for understanding the relative importance of adaptive and maladaptive gene expression responses to herbicide. Specifically, we compare leaf gene expression changes caused by herbicide to the expression changes that evolve in response to artificial selection for herbicide resistance. We identify a number of genes that show plastic and evolved responses to herbicide and find that for the majority of genes with both plastic and evolved responses, plastic responses appear to be adaptive. We also find that selection for herbicide response increases gene expression plasticity. Overall, these results show the importance of adaptive plasticity for herbicide resistance in a common weed and that expression changes in response to strong environmental change can be adaptive.
\end{abstract}

\section{Impact statement}

Predicting whether and how organisms will adapt to environmental change is a crucial goal. However, this goal can be complicated because environmental change can alter traits, in a process called plasticity. The extent and fitness consequences of plasticity will have important effects on the adaptive process. In this study, we use adaptation to herbicide in the agricultural weed, the common morning glory, as a model for understanding the extent and fitness consequences of plasticity in gene expression. We find evidence that gene expression plasticity is adaptive in the presence of herbicide, suggesting that understanding plasticity is crucial for understanding how organisms adapt to new environments.

\section{Introduction}

Determining the fitness consequences of plastic responses is crucial for predicting response to selection [1], understanding the maintenance of variation for phenotypes [2], and breeding plants for new, challenging environments $[3,4]$. Theoretical and empirical evidence show that environments that fluctuate predictably in such a way that there is no optimum phenotype across time will favor adaptive plasticity $[5,6]$. Plastic responses that reduce fitness, or 'maladaptive plasticity', can occur when stressful environments overwhelm organisms' ability to maintain fitness [5] or new environments expose cryptic variation, although cryptic variation can also be beneficial [7]. The presence of adaptive plasticity can allow populations to persist 
in the face of stressful conditions, [8] and either reduce the strength of selection by masking additive genetic variation or contribute to adaptation in novel environments [9]. Whether or not adaptive plasticity will facilitate or constrain genetic responses to selection depends on how close the plastic response gets individuals to the optimum phenotype [5]. However, despite the clear importance of disentangling adaptive from maladaptive plasticity, we lack a clear view of how the plastic responses affect fitness [5,10-12]. In particular, despite hypotheses and evidence that gene expression changes underlie many plastic responses in traditional phenotypes, the fitness consequences of gene expression plasticity are unknown $[13,14]$.

A number of approaches have been used to describe the fitness consequences of plasticity. For example, some studies have measured fitness in organisms where a specific plastic response has or has not been induced to evaluate whether the plastic response increases fitness in the environments that induce it $[15,16]$. However, many plastic responses, including expression level, are not amenable to these types of manipulations. An alternative approach to understanding the fitness consequences of plasticity is comparing the fitness of genetically distinct individuals that exhibit different levels of plasticity, if thereis natural genetic variation for plasticity [17-21]. In this study we focus on an alternative approach that allows us to specifically investigate gene expression variation, since it is a crucial component of plastic responses.

Here, we determine whether plastic changes in gene expression are adaptive or maladaptive by comparing plastic expression changes elicited by an environment with the expression changes that evolve during adaptation to that environment. If plasticity is adaptive and increases fitness in the new environment, the direction of plastic responses is expected to match the direction of evolved responses. Alternatively, if plasticity is maladaptive, we expect plastic responses to be opposite that of adaptive responses. Previous applications of this approach have found that plastic expression changes tend to be maladaptive in guppy brain expression response to predation environments [22] and Drosophila gene expression responses to diet [23]. An analysis of the previously mentioned guppy study along with studies from Escherichia coli and yeast also found a preponderance of maladaptive expression plasticity [24,25]. Strikingly, this approach has not been widely applied beyond these few studies.

We use artificially evolved glyphosate-resistant lineages of the common morning glory, Ipomoea purpurea, as a model for examining the fitness consequences of plastic changes in gene expression. There is a long history of using pesticide resistance evolution as models for adaptation $[26,27]$ and the fitness costs of adaptation [27-29] but there is a significant gap in our understanding of the role of plasticity inshaping resistance evolution. Resistance to glyphosate (i.e., the active ingredient in the herbicide RoundUp) can involve either 'target-site' or 'non-target site' mutations [30]. In the former case, the genes that are the target of the herbicide contain mutations that lead to resistance whereas in the latter case any other gene that is not the target of the herbicide may be involved [27]. Non-target site resistance can be a subset of generalplastic responses to abiotic stress and often involves multiple genes [31].

The common morning glory provides an excellent study system for examining questions about the role of adaptive versus maladaptive plasticity on the process of herbicide resistance evolution. Natural populations of I. purpurea vary in the level of glyphosate resistance [32], with non-target site herbicide resistance the most likely explanation for resistance in this species [33]. There is strong evidence of fitness costs associated with resistance in I. purpurea, which is consistent with the idea that resistance incurs a trade-off $[34,35]$. However, the specific role of plasticity in resistance evolution in I. purpurea, or any other weedy species is unknown.

In this study, we used seeds from an experimental evolution experiment designed to select for increased herbicide resistance in plants descending from a single population [35]. Unlike previous studies that 
used this approach, the population these plants were collected from did occasionally experience the new environment, herbicide treatment and displayed additive genetic variation for resistance. However, despite past experience of herbicide, artificial selection for resistance did successfully increase survival in herbicide treatments [33]. We compared leaf transcriptomes of plants from the experimentally evolved resistant lineages and from control, non-evolved lineages that were exposed to glyphosate along with replicates of each selection line that were not treated with the herbicide. We used these transcriptomes to quantify how often plastic expression responses to glyphosate aligned with the expression changes that evolved during selection for resistance. We also investigated whether selection in the herbicide environment favored increased plasticity. Overall, our results demonstrate a preponderance of adaptive gene expression plasticity in response to herbicide and that selection for increased resistance increases plasticity.

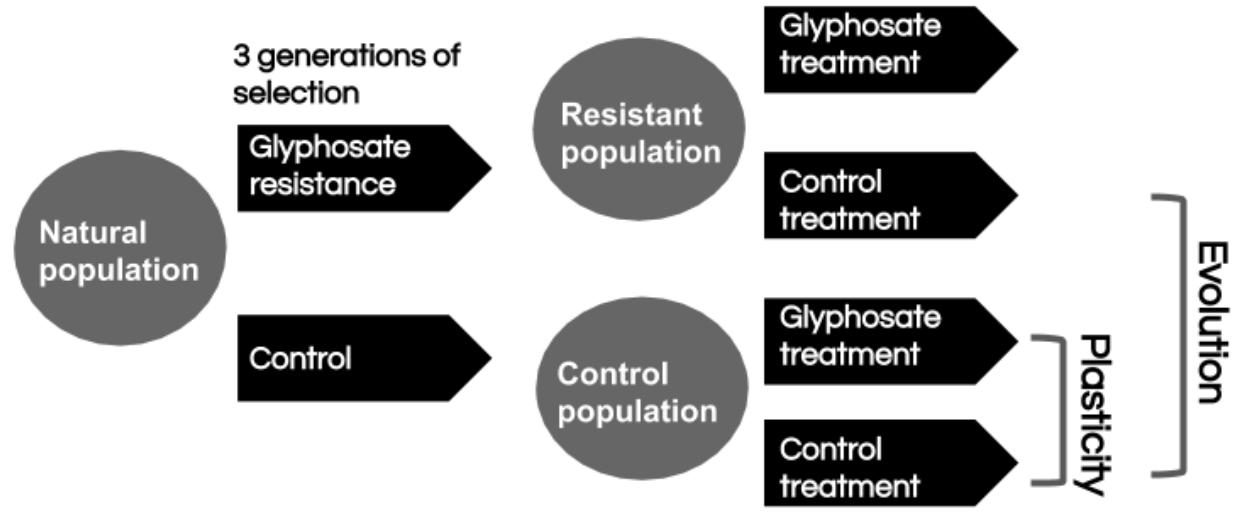

Figure 1: Conceptual figure of the experimental design. This figure shows the breeding scheme that was used to generate the plants used in this study. Plastic expression changes were measured by comparing gene expression in control plants from a control treatment and from the glyphosate treatment. Evolved expression changes were used by comparing control treatment plants from the control and resistance populations.

\section{Methods}

Study system

Ipomoea purpurea (L.) Roth (Convolvulaceae) is a short-lived annual vine with a relatively rapid generation time (from planted seed to producing seed within six weeks [36]) that is typically found growing on roadsides and in agricultural settings or areas of high disturbance [37]. Native to the central highlands of Mexico [38], this noxious invasive is found in every state within the US but it is particularly troublesome in the agricultural fields within the southeast and Midwestern US [32]. It has a mixed-mating system (average outcrossing rate $=0.5$ ) with outcrossing rates that vary from highly selfing to highly outcrossing across populations [39].

\section{Genetic material}


All plants used in the experiment descended from a single population at the previous site of the University of Georgia Plant Sciences Farm in Oconee, GA in 2000. We haphazardly sampled seeds from 122 maternal individuals at approximately every 1 meter on a transect in this population. The offspring of this sample (Generation 0 or G0) were screened for high or low glyphosate resistance in a greenhouse [40] and the offspring of the top $20 \%$ highly resistant lines (24 families) were used to be the parents for two resistant selection lines (12 parents each) and 24 parents were randomly chosen from the whole population to be the parents of two control lines. Individuals from each set of parents were grown up (Generation 1 or G1) and another generation of artificial selection was performedin the resistant selection lines using a "family selection" design by propagating siblings of the individuals in the top $20 \%$ of herbicide resistance in the population. Random individuals within each control line were chosen for the next generation to generate Generation 2 or G2 seeds. Another identical round of selection was performed on the G2 individuals to generate G3 progeny. In all crosses, individuals were used as both the pollen and ovule parent. See [35] for additional details. In a field trial, G3 plants from the resistant lines had higher survival than control lines after herbicide treatment [35].

After the field screening of G3 seeds, the 3 mostresistant families were chosen from each of the selection lines and seeds from the G2 parents of these six families were crossed to each other (across lines) to generate outcrossed seeds and crossed to themselves to generate selfed seeds. The same procedure was used to generate seeds from the G2 control parents.

\section{Experiment}

We planted 20 replicate seeds from each maternal line into two blocks and two treatments (herbicide vs no herbicide) in a randomized block design in a fenced one acre agricultural field at the Matthaei Botanical Gardens (MBGNA) at the University of Michigan in the spring of 2015. There are no natural I. purpurea individuals anywhere on or surrounding MBGNA. Throughout the experiment, we watered plants when needed to prevent wilting. After 6 weeks of growth, we applied $0.84 \mathrm{~kg}$ ai $/$ ha glyphosate (slightly higher than $1 / 2$ the suggested field dose of $1.54 \mathrm{~kg}$ ai $/ \mathrm{ha}$ ) to the plants in the herbicide treatment with a $\mathrm{CO}_{2}$ sprayer. We used a relatively low level of herbicide to cause stress but avoid killing the plants.

We collected leaf tissue from seeds at two time points post-herbicide application: 8 and 32 hours after spraying. Within each time point we randomly chose two individuals per family (one replicate individual generated from selfing and one from outcrossing per maternal line) and froze 1-2 young leaves in liquid nitrogen. All tissue at a given time point was collected and frozen within one hour.

\section{Transcriptome data generation}

We extracted RNA from leaf tissue with Qiagen RNAeasy kits. We individually indexed libraries using the llumina TRUseq96 indexer mRNA stranded kit. Pooled libraries were run on 7 lanes of 50bp, single end sequencing on the Illumina HiSeq 4000 resulting in an average of 28 million reads per individuals.

We aligned single-end (1x52nt) adapter-trimmed Illumina RNA-seq reads separately for all samples to the Ipomoea purpurea v 2.0 genome [41] using the splice aware STAR aligner in its basic two-pass mode [42]. Genome annotation for STAR was generated using GATK's CreateSequenceDictionary (v4, [43]), samtools' v1.3 faidx function [44] and STAR's genomeGenerate option together with an Augustus-derived GFF3 annotation [45] provided with the v.2.0 assembly. The resulting sorted bam alignment files were used for measuring RNA-seq digital gene expression using the provided GFF3 gene annotation and the featureCounts tool in the SubRead package [46]. 
Homologs in A. thaliana were found based on protein homology (TAIR10 v.20101214, https://www.arabidopsis.org/download_files/Proteins/TAIR10_protein_lists/TAIR10_pep_20101214), with the blastp mode of blastall (NCBI v.2.2.26) used to find the closest matching thaliana protein to each Augustus-predicted I. purpurea protein sequence with an upper limit p-value for reporting a homolog of 1e-6.

\section{Differential expression analysis}

We used Edgr's filterByExpr function with the default settings (minimum 10 counts-per-million per gene) to filter the gene set to 25,534 genes [47] We calculated normalization factors that scale each sample by raw library size with Edgr's calcNormFactors function [48-50]. Read counts were transformed with the voom function in Limma and we used Limma to run linear models that estimated differential expression between samples. [51-53].

We conducted the following comparisons for differential expression:

1. Identifying genes with plastic responses by comparing non-selected control lines in herbicide spray ( $\mathrm{n}=16)$ to non-selected control lines not sprayed with herbicide $(\mathrm{n}=15)$. This comparison identifies plasticity in the original population before selection but note that these lines were grown in lab conditions for three generations, so drift or lab selection could affect our observations of plasticity. We also conducted these comparisons within the two timepoints tested, so we compared leaves collected 8 hours after spray $(n=8)$ to leaves from unsprayed plants collected at the same time $(n=8)$ and leaves collected 32 hours after spray $(n=8)$ to leaves from unsprayed plants collected at the same time $(n=7)$.

2. Identifying genes with evolved responses after selection for herbicide resistance by comparing resistance selection lines not sprayed $(n=15)$ to original lines not sprayed $(n=15)$.

3. Identifying genes with evolved expression responses in the herbicide environment after selection for herbicide resistance by comparing resistance selection lines in spray conditions $(n=15)$ to original lines in spray conditions $(n=15)$.

We conducted GO enrichment with PANTHER on pantherdb.org [54] using the Fisher's exact test with a false discovery rate analysis. We compared genes that had increased expression in herbicide $(3,789$ annotated genes) or decreased expression ( $\mathrm{N}$ annotated genes) to all expressed genes from the sample (12,702 annotated genes).

All code is available at https://github.com/emjosephs/morning-glory and archived at

\section{Results}

\section{Plastic and evolved expression responses to herbicide}

We measured plastic responses to herbicide in control plants that had not been artificially selected for herbicide resistance (Figure 1). Morning glory leaf gene expression responded plastically to herbicide, both 8 and 32 hours after herbicide application. Out of the 27,037 genes tested, 3,519 genes had higher expression and 2,841 genes had lower expression 8 hours after spray (FDR $<0.1$ ) and 5,732 genes had higher expression and 5,285 genes had lower expression 32 hours after spray (FDR $<0.1$ ). The log fold change of expression changes caused by spray was correlated across the 8 and 32 hour measurements (cor coeff $=0.614, p<0.001$, Figure S1). There were only 137 genes with significant (FDR $<0.1)$ plastic responses to spray at both time 
points where the direction of response differed between the 8 hour and 32 hour time points. Because of the similarity of response at 8 and 32 hours after spray, we pooled data for future analyses.

When data from both time points was pooled to estimate plastic responses to spray in general, 5,734 genes had lower expression in response to pesticide spray and 6,171 had increased expression in response to spray (FDR $<0.1$, Figure 2). Genes with increased expression after spray were enriched for a number of GO biological processes at FDR $<0.01$, including a number of terms related to abiotic and biotic interactions (Table S1). In contrast, genes with reduced expression after spray were enriched for GO terms related to photosynthesis (Table S2).

Gene expression in I. purpurea leaves responded to selection for resistance (Figure 2). After three generations of selection for herbicide resistance, 166 genes showed decreased expression and 133 genes showed increased expression (FDR $<0.1$, Figure 2A). These genes were not significantly enriched for any GO terms.
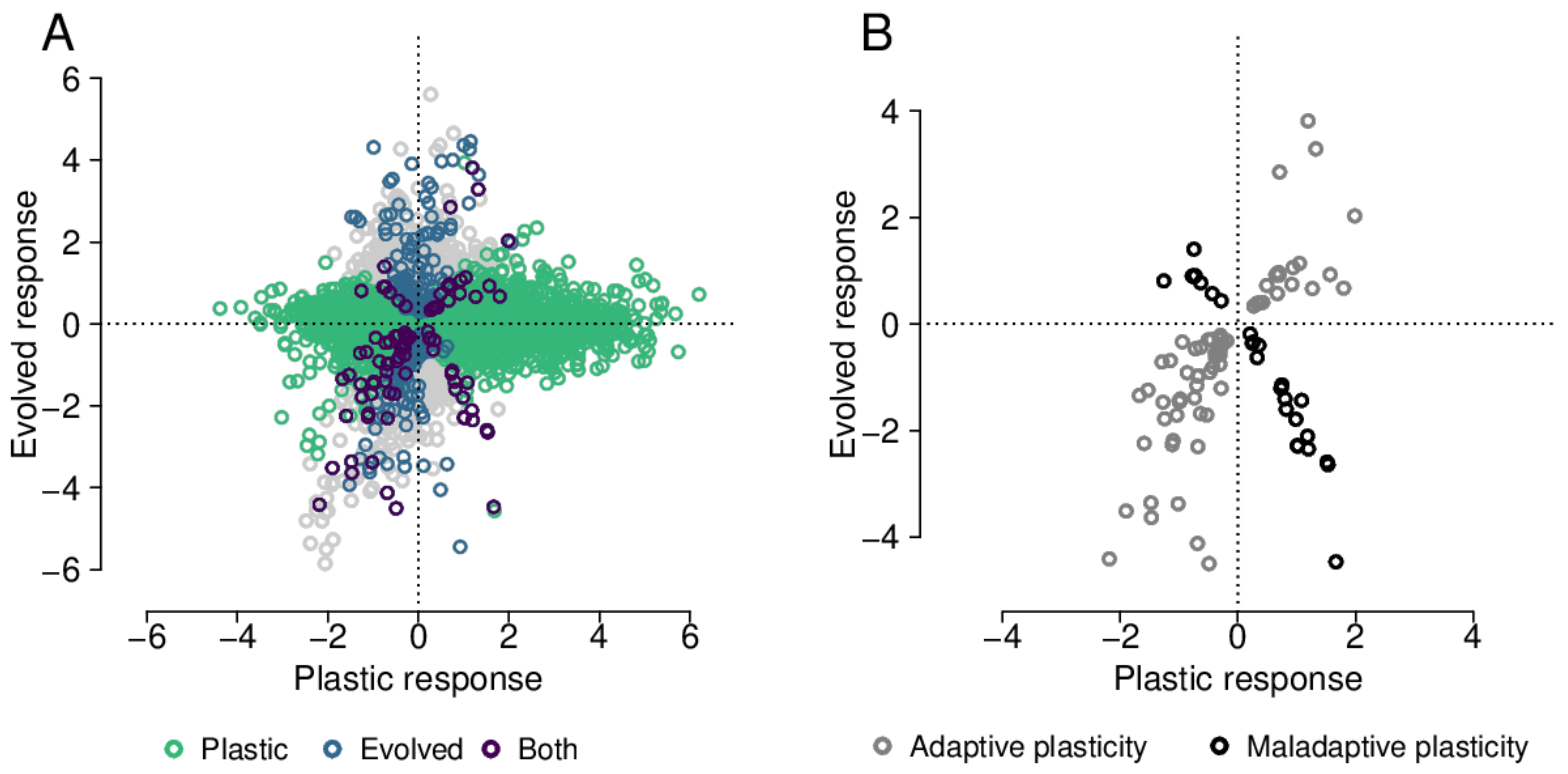

Figure 2: Plastic and evolved changes in expression: A) The $x$ axis shows plastic responses to glyphosate, specifically the log fold change in expression between sprayed and nonsprayed conditions where positive values indicate increased expression in herbicide spray. The y axis shows the log fold difference in expression between lines selected for increased resistance to herbicide and lines that were not selected ('original lines'), where positive values indicate increased expression in the resistance selection lines. Each point represents one gene, colored by whether they have significant $(\mathrm{FDR}<0.1)$ plastic expression responses, evolved expression responses or both. B) The same as (A), but only genes with significant responses to selection and treatment are shown.

\section{The prevalence of adaptive and maladaptive plasticity}

There were 94 genes that had both significant plastic responses to spray and evolved responses to selection for resistance in unsprayed conditions (FDR $<0.1)$. Within these 94 genes, 68 had plastic responses in the same direction as evolved responses, consistent with adaptive plasticity (Binomial $\mathrm{p}=1.7 \times 10^{-5}$, Figure 2B). Overall, with the majority of genes showing plastic responses in the same direction as evolved responses, these results show that more of the expression responses in response to herbicide are adaptive than 
maladaptive. We only looked at selection in non-sprayed conditions to avoid confounding selective response and plasticity [55].

The pattern of adaptive plasticity was also evident for gene expression in samples collected at 8 hours after spray, where 44 out of 56 genes with plastic and evolved responses showed adaptive plasticity (binomial $\mathrm{p}=2 \times 10^{-5}$ ), and marginally significant at 32 hours after spray, where 44 of 71 genes with significant adaptive and plastic responses exhibited adaptive plasticity (binomial $p=0.057$, Figure S2A, B). While there were more genes that showed evidence of either maladaptive or adaptive plasticity 32 hours after spray, the directionality of the response were broadly consistent, with only one of the genes changing direction from adaptive after 8 hours to maladaptive after 32 hours.

\section{Specific genes showing adaptive and maladaptive plasticity for expression}

It was possible to annotate 41 of the 68 genes with evidence of adaptive plasticity based on homology to Arabidopsis thaliana (Table S3). Potential genes of interest included the homolog of ATR7, which is associated with oxidative stress tolerance [56] and PRL1, which is associated with growth and immunity [57]. However, many genes identified as having adaptive plasticity did not have homologs with obvious links to processes important for herbicide or other stress responses (Table S3).

We were able to identify homologs from $A$. thaliana for 24 of the 26 genes with evidence of maladaptive plasticity. Many of the genes that show increased plastic expression during spray but decreased expression after selection for resistance were homologs of three $A$. thaliana leucine-rich repeat protein kinase family proteins (Figure 3, Table S3). Leucine-rich repeat protein kinases are often involved in responses to stress [58-61]. Many of these genes were located neareach other in the I. purpurea reference genome, suggesting that they are recent tandem duplicates. The observed patterns of expression changes suggest that selection for herbicide resistance reduced the expression of these genes, providing a potential future path for understanding the mechanisms of adaptation to herbicides.

\section{Selection for herbicide resistance increases plasticity.}

If the plastic expression changes that occur in response to spray are adaptive, selection for increased herbicide resistance should increase the extent and magnitude of expression changes in response to herbicide. Consistent with this prediction, more genes had plastic expression responses to herbicide after selection for resistance compared to control lines (FDR $<0.1$, Figure 3A). Similarly, 59\% of all genes studied had a greater expression response to herbicide after selection for resistance than in control lines (Figure 3B). However, across all genes, plastic responses to selection were correlated in control and resistant lines, suggesting that selection for resistance did not drastically alter patterns of plasticity (Figure 3C, Figure S3). 

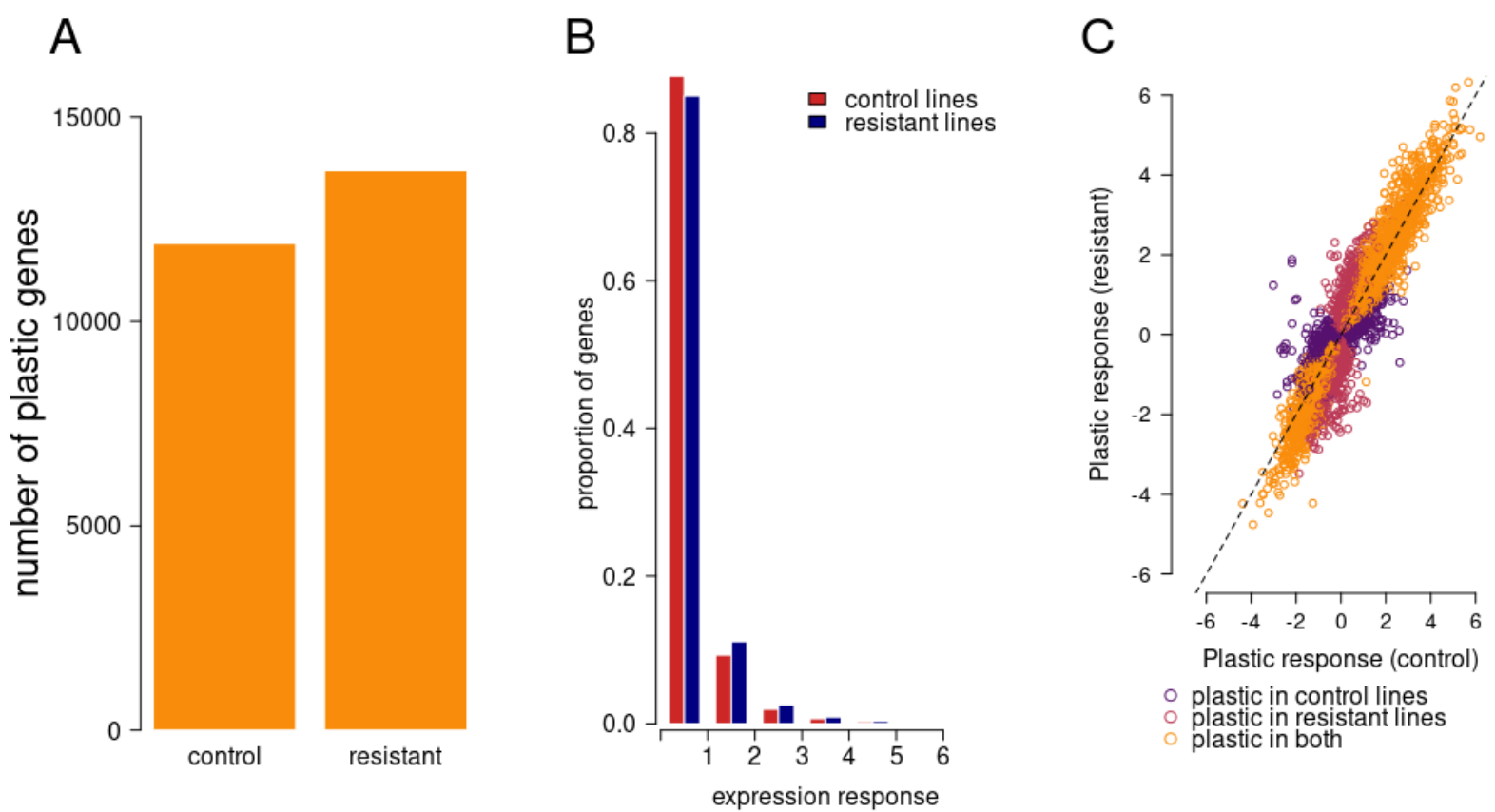

Figure 3: A) The number of genes showing plastic expression differences in response to herbicide at FDR $<0.1$ in control and selected lines B) A histogram of gene expression responses to herbicide, measured as the absolute value of the log 2 fold change of expression measured with and without herbicide. C) Plastic expression in control lines and lines selected for resistance. Response is shown on a log2 scale and the $x=y$ line is plotted with a dashed black line. Points are colored by whether they show a significant plastic response to spray in resistant and control lines. Genes without a significant response in either set of lines are not shown.

\section{Discussion}

Here, we have shown that the gene expression plasticity of Ipomoea purpurea after glyphosate application shows patterns consistent with being adaptive. The plastic gene expression changes we identified generally align in the same direction as evolved changes after artificial selection for increased herbicide resistance. These results differ from those of other systems, where maladaptive gene expression plasticity appears to be more common [22-25]. There are a number of potential explanations for this difference. First, the population studied here may exist in a set of conditions where plasticity is predicted to be adaptive $[1,8,62,63]$. For example, the ancestors of the lines used in this study were collected in 2000 , after experiencing intermittent glyphosate application for at least 8 years [64], so fluctuating selection from herbicide use may have selected for plastic responses to herbicide. In addition, tolerance and resistance to glyphosate were present in I. purpurea before the use of glyphosate, suggesting that these plastic traits may be pleiotropic with plastic responses to other stresses [65]. While in agricultural settings, herbicide is generally applied once per generation, the timing of application could vary year-by-year relative to weed developmental timing. If the physiological effects of herbicidediffer across development, this treatment could contribute to fluctuating selection, a process that can select for adaptive plasticity [5].

There are a number of potential consequences of widespreadadaptive plasticity. Adaptive plasticity could facilitate adaptation to new conditions by allowing populations to persist long enough for adaptation to occur but it could also hinder local adaptation by masking genetic variation from selection [5]. Which process 
occurs depends on how close the plastic response is to the phenotypic optimum [5,8]. In this study, despite evidence of potential adaptive gene expression plasticity, the population was still able to evolve increased resistance to herbicide [35], suggesting that the plastic responses do not completely move plants to the optimum trait value. Interestingly, the results here suggest that further adaptation to herbicide treatment increased the number of genes with expression plasticity and the magnitude of this plasticity.

It is unclear if and how the results of this studywould change if evolutionary changes in expression were evaluated in natural populations that have evolved resistance instead of in artificially selected populations. The responses to selection that occur in experimental evolution will differ from those in natural populations [66]. An additional limitation for all studies in non-model systems is that we are reliant on GO terms for homologs in Arabidopsis thaliana, a distantly related species. A lack of gene or functional conservation will erode our ability to make conclusions about gene function from this data.

There are also limitations to using leaf gene expression as a trait in studies of plasticity. First, leaves are made up of multiple tissues, so variation in different tissue types, instead of direct increases in the transcription of genes within cells, could drive changes in gene expression [67]. Second, the expression level of different genes is not independent and observations of many genes changing in expression could stem from one or a few trans-regulatory factors [68]. Incorporating information about how regulatory networks respond to environmental changes and stresses will be an important next step to understanding the links between selection, plasticity, and adaptation to stressful environments.

This is the first paper to directly investigate the adaptive potential of plastic expression changes in response to herbicide. Previous work has found evidence of gene expression changes in response to herbicide, often in genes not previously known to be important for herbicide response [69-74], but these studies did not directly investigate whether plastic responses appeared to be adaptive. One hint at the importance of maladaptive plasticity comes from the observation that a susceptible population of Amaranthus tuberculatus increased rapid expression responses to herbicide compared to a naturally resistant population [75], suggesting that plastic expression changes in response to herbicide could be maladaptive in this species. If our work using an artificially evolved population is any guide for resistance evolution in natural populations, it suggests that selection for increased resistance may reduce expression of some genes, perhaps those with environment-sensing functions (e.g. leucine rich repeat protein kinases), while also leading to increased plasticity in other genes.

In summary, we have shown that I. purpurea gene expression responds plastically to herbicide application and evolutionarily to selection for resistance. Crucially, plastic expression responses generally go in the same direction as evolved responses, consistent with the idea that this plasticity is adaptive. We also showed that consistent selection for resistance can increase plasticity, suggesting plasticity is a key component of herbicide resistance in I. purpurea. All together, this work demonstrates the importance of adaptive plasticity for the evolution of resistance to environmental stressors.

\section{Acknowledgements}

We thank Jim Leebens-Mack for help with assembly and annotation of the I. purpurea reference genome, McKena Wilson and Maya Wilson Brown for research assistance, and Yuheng Huang, Matt Osmond, John Stinchcombe, Sophie Buysse, the Whitehead lab, and two anonymous reviewers for helpful suggestions. This work was funded by Michigan State University, the University of Michigan, and USDA NIFA grants 24892 \& 28497 to RSB. 


\section{Supplemental figures}

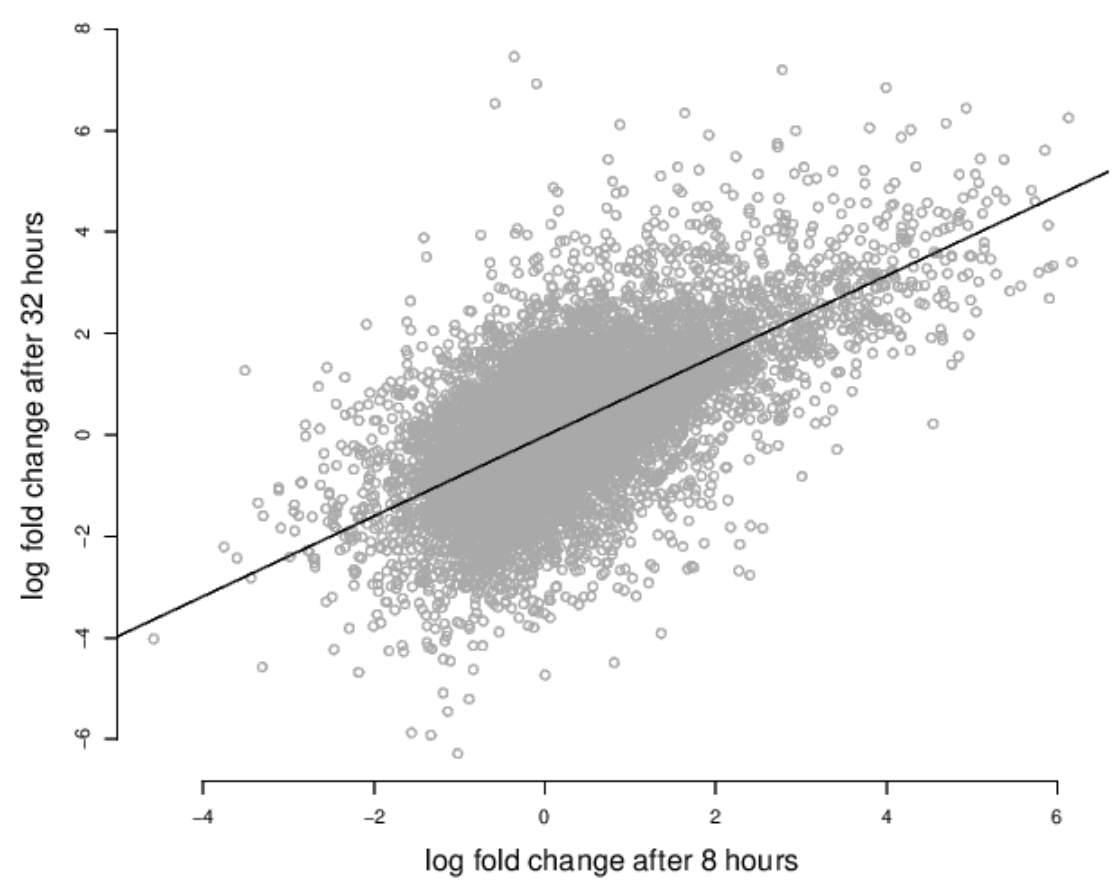

Figure S1: Plastic responses to herbicide spray 8 and 32 hours after treatment. The $x$ axis shows the log-fold difference in expression in sprayed and unsprayed conditions 8 hours after treatment and the $y$ axis shows the same value 32 hours after treatment. The black dotted line shows a line with a slope of 1 and intercept of 0 . The correlation coefficient is 0.616 . All genes are shown. 


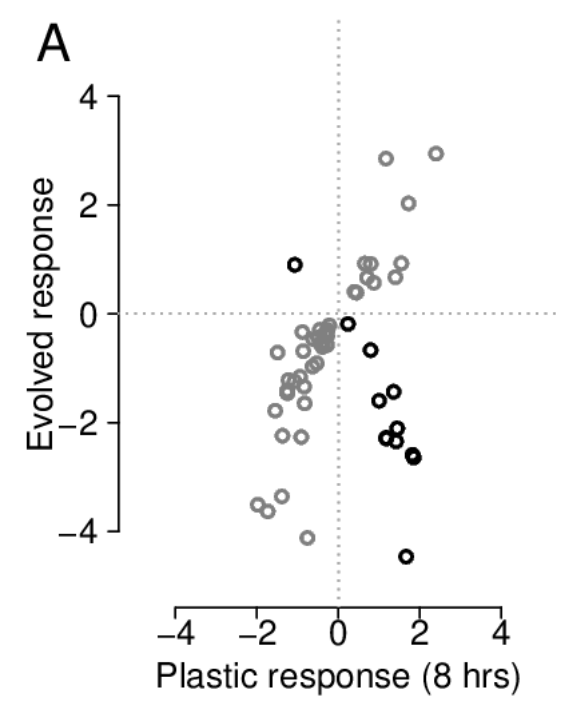

- Adaptive plasticity

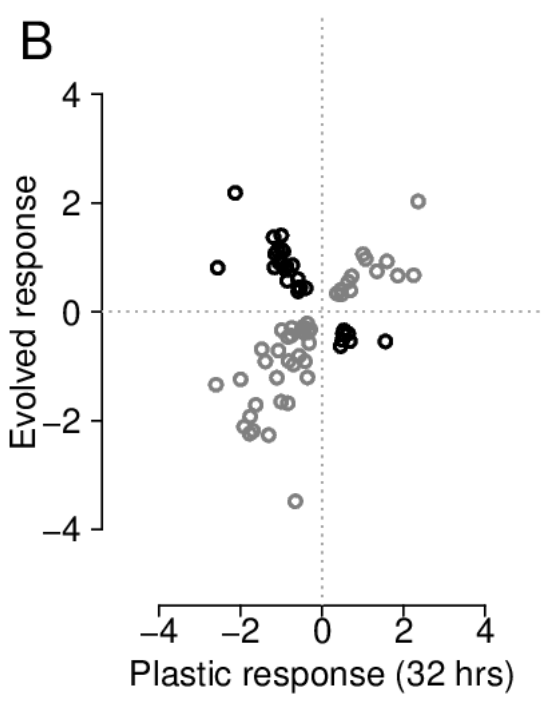

- Maladaptive plasticity

Figure S2: Adaptive and maladaptive expression plasticity at 8 hours (A) and 32 hours (B) after herbicide treatment. In both panels, the $x$ axis shows plastic responses to glyphosate, specifically the log fold change in expression between sprayed and nonsprayed conditions where positive values indicate increased expression in herbicide spray. The y axis shows the log fold difference in expression between lines selected for increased resistance to herbicide and lines that were not selected ('original lines'), where positive values indicate increased expression in the resistance selection lines. Each point represents a gene and all genes where there was both a significant plastic and an evolved response are shown $($ FDR $<0.1)$. 
bioRxiv preprint doi: https://doi.org/10.1101/2020 09.25.313650; this version posted May 3, 2021. The copyright holder for this preprint (which was not certified by peer review) is the author/funder, who has granted bioRxiv a license to display the preprint in perpetuity. It is made available under aCC-BY-NC 4.0 International license.

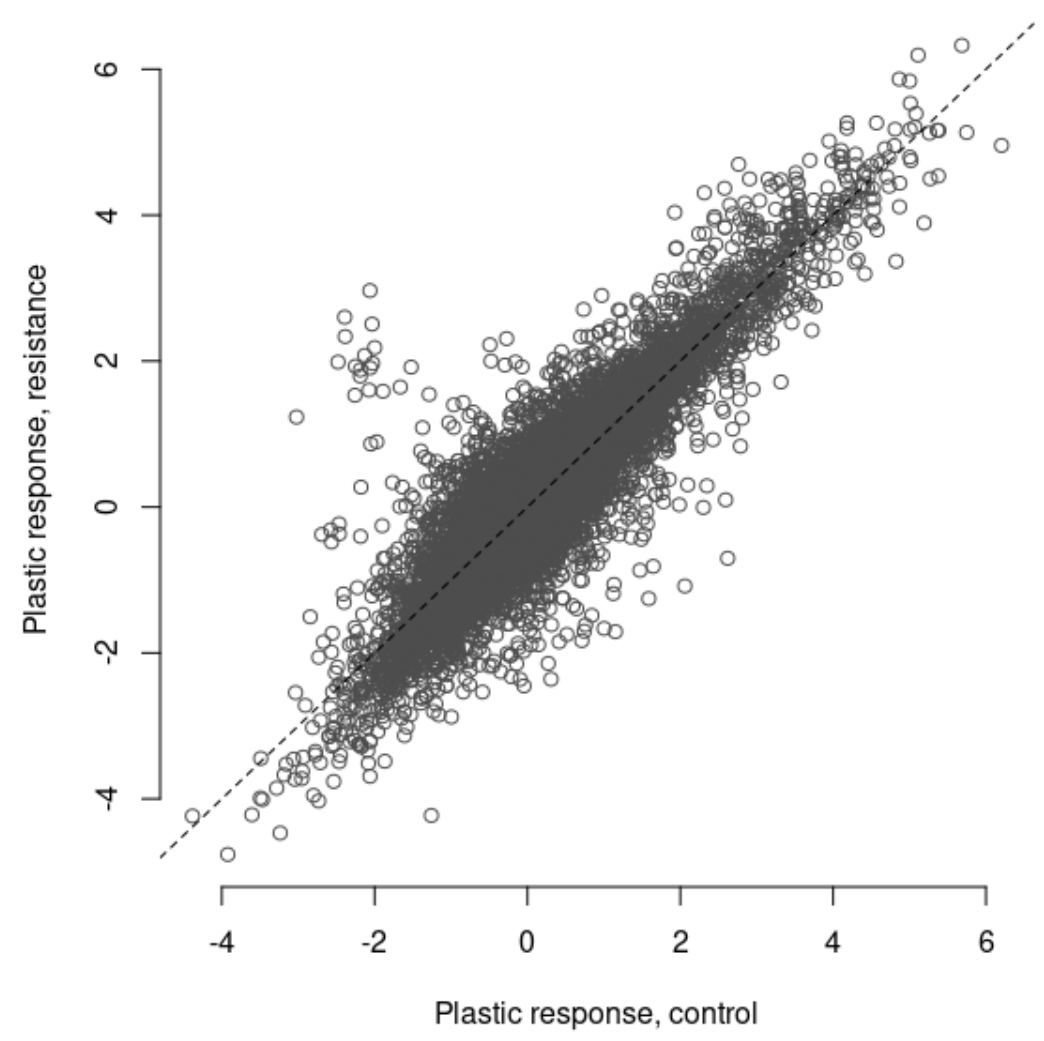

Figure S3: Plastic expression changes in control and resistance lines for all genes. Each point represents one gene, and the $y=x$ line is plotted with a dashed line. 


\section{References}

1. Reed TE, Waples RS, Schindler DE, Hard JJ, Kinnison MT. Phenotypic plasticity and population viability: the importance of environmental predictability. Proc Biol Sci. 2010;277: 3391-3400.

2. Josephs EB. Determining the evolutionary forces shaping $G \times$ E. New Phytol. 2018;219: 31-36.

3. Gage JL, Jarquin D, Romay C, Lorenz A, Buckler ES, Kaeppler S, et al. The effect of artificial selection on phenotypic plasticity in maize. Nat Commun. 2017;8: 1348.

4. Kusmec A, de Leon N, Schnable PS. Harnessing Phenotypic Plasticity to Improve Maize Yields. Front Plant Sci. 2018;9: 1377.

5. Ghalambor CK, McKay JK, Carroll SP, Reznick DN. Adaptive versus non-adaptive phenotypic plasticity and the potential for contemporary adaptation in new environments. Funct Ecol. 2007;21: 394-407.

6. Leung C, Rescan M, Grulois D, Chevin L-M. Reduced phenotypic plasticity evolves in less predictable environments. Ecol Lett. 2020. doi:10.1111/ele.13598

7. Paaby AB, Rockman MV. Cryptic genetic variation: evolution's hidden substrate. Nat Rev Genet. 2014;15: 247-258.

8. Price TD, Qvarnström A, Irwin DE. The role of phenotypic plasticity in driving genetic evolution. Proc Biol Sci. 2003;270: 1433-1440.

9. Chevin L-M, Lande R. WHEN DO ADAPTIVE PLASTICITY AND GENETIC EVOLUTION PREVENT EXTINCTION OF A DENSITY-REGULATED POPULATION? Evolution. 2010;64: 1143-1150.

10. Schlichting CD. The Evolution of Phenotypic Plasticity in Plants. Annu Rev Ecol Syst. 1986;17: 667-693.

11. Bradshaw AD. Evolutionary significance of phenotypic plasticity in plants. Adv Genet. 1965;13: $115-155$.

12. Via S, Gomulkiewicz R, De Jong G, Scheiner SM, Schlichting CD, Van Tienderen PH. Adaptive phenotypic plasticity: consensus and controversy. Trends Ecol Evol. 1995;10: 212-217.

13. Hodgins-Davis A, Townsend JP. Evolving gene expression: from $G$ to $E$ to $G \times E$. Trends Ecol Evol. 2009;24: 649-658.

14. Lovell JT, Schwartz S, Lowry DB, Shakirov EV, Bonnette JE, Weng X, et al. Drought responsive gene expression regulatory divergence between upland and lowland ecotypes of a perennial C4 grass. Genome Res. 2016. doi:10.1101/gr.198135.115

15. Dudley SA, Schmitt J. Testing the Adaptive Plasticity Hypothesis: Density-Dependent Selection on Manipulated Stem Length in Impatiens capensis. Am Nat. 1996;147: 445-465.

16. Schmitt J, Dudley SA, Pigliucci M. Manipulative Approaches to Testing Adaptive Plasticity: 
Phytochrome-Mediated Shade-Avoidance Responses in Plants. Am Nat. 1999;154: S43-S54.

17. Wagner MR, Mitchell-Olds T. Plasticity of plant defense and its evolutionary implications in wild populations of Boechera stricta. Evolution. 2018;72:1034-1049.

18. Weis AE, Gorman WL. Measuring Selection on Reaction Norms: An Exploration of the Eurosta-solidago System. Evolution. 1990;44: 820-831.

19. Baythavong BS, Stanton ML. Characterizing selection on phenotypic plasticity in response to natural environmental heterogeneity. Evolution. 2010;64: 2904-2920.

20. Stinchcombe JR, Dorn LA, Schmitt J. Flowering time plasticity in Arabidopsis thaliana: a reanalysis of. J Evol Biol. 2004;17: 197-207.

21. Scheiner SM, Callahan HS. MEASURING NATURAL SELECTION ON PHENOTYPIC PLASTICITY. Evolution. 1999;53: 1704-1713.

22. Ghalambor CK, Hoke KL, Ruell EW, Fischer EK, Reznick DN, Hughes KA. Non-adaptive plasticity potentiates rapid adaptive evolution of gene expression in nature. Nature. 2015;525: 372-375.

23. Huang Y, Agrawal AF. Experimental Evolution of Gene Expression and Plasticity in Alternative Selective Regimes. PLoS Genet. 2016;12: e1006336.

24. Ho W-C, Zhang J. Evolutionary adaptations to new environments generally reverse plastic phenotypic changes. Nat Commun. 2018;9: 350.

25. Ho W-C, Zhang J. Genetic gene expression changes during environmental adaptations tend to reverse plastic changes even after the correction for statistical nonindependence. Mol Biol Evol. 2019. doi:10.1093/molbev/msz002

26. Ffrench-Constant RH. The molecular genetics of insecticide resistance. Genetics. 2013;194: 807-815.

27. Baucom RS. Evolutionary and ecological insights from herbicide-resistant weeds: what have we learned about plant adaptation, and what is left to uncover? New Phytol. 2019;223: 68-82.

28. Bergelson J, Purrington CB. Surveying Patterns in the Cost of Resistance in Plants. Am Nat. 1996;148: $536-558$.

29. Ffrench-Constant RH, Bass C. Does resistance really carry a fitness cost? Curr Opin Insect Sci. 2017;21: $39-46$.

30. Powles SB, Yu Q. Evolution in action: plants resistant to herbicides. Annu Rev Plant Biol. 2010;61: $317-347$.

31. Délye C, Jasieniuk M, Le Corre V. Deciphering the evolution of herbicide resistance in weeds. Trends Genet. 2013;29: 649-658.

32. Kuester A, Chang S-M, Baucom RS. The geographic mosaic of herbicide resistance evolution in the common morning glory, Ipomoea purpurea: Evidence for resistance hotspots and low genetic differentiation across the landscape. Evol Appl. 2015;8: 821-833. 
33. Van Etten ML, Soble A, Baucom RS. Variable inbreeding depression may explain associations between the mating system and herbicide resistance in the common morning glory. Mol Ecol. 2021. doi: $10.1111 /$ mec.15852

34. Van Etten ML, Kuester A, Chang S-M, Baucom RS. Fitness costs of herbicide resistance across natural populations of the common morning glory, Ipomoea purpurea. Evolution. 2016;70: 2199-2210.

35. Debban CL, Okum S, Pieper KE, Wilson A, Baucom RS. An examination of fitness costs of glyphosate resistance in the common morning glory, Ipomoea purpurea. Ecol Evol. 2015;5: 5284-5294.

36. Baucom RS, Chang S-M, Kniskern JM, Rausher MD, Stinchcombe JR. Morning glory as a powerful model in ecological genomics: tracing adaptation through both natural and artificial selection. Heredity . 2011;107: 377-385.

37. Baucom RS, Mauricio R. Constraints on the evolution of tolerance to herbicide in the common morning glory: resistance and tolerance are mutually exclusive. Evolution. 2008;62: 2842-2854.

38. Fang Z, Gonzales AM, Durbin ML, Meyer KKT, Miller BH, Volz KM, et al. Tracing the geographic origins of weedy Ipomoea purpurea in the southeastern United States. J Hered. 2013;104: 666-677.

39. Kuester A, Fall E, Chang S-M, Baucom R. Shifts in outcrossing rates and changes to floral traits are associated with the evolution of herbicide resistance in the common morning glory. bioRxiv. 2016. p. 061689. doi:10.1101/061689

40. Baucom RS, Mauricio R. The evolution of novel herbicide tolerance in a noxious weed: the geographic mosaic of selection. Evol Ecol. 2008. Available:

https://link.springer.com/content/pdf/10.1007/s10682-007-9160-1.pdf

41. Gupta S, Harkess A, Soble A, Van Etten M, Leebens-Mack J, Baucom RS. Inter-chromosomal linkage disequilibrium and linked fitness cost loci influence the evolution of nontarget site herbicide resistance in an agricultural weed. bioRxiv. 2021. p. 2021.04.04.438381. doi:10.1101/2021.04.04.438381

42. Dobin A, Davis CA, Schlesinger F, Drenkow J, Zaleski C, Jha S, et al. STAR: ultrafast universal RNA-seq aligner. Bioinformatics. 2013;29: 15-21.

43. McKenna A, Hanna M, Banks E, Sivachenko A, Cibulskis K, Kernytsky A, et al. The Genome Analysis Toolkit: a MapReduce framework for analyzing next-generation DNA sequencing data. Genome Res. 2010;20: 1297-1303.

44. Li H, Handsaker B, Wysoker A, Fennell T, Ruan J, Homer N, et al. The Sequence Alignment/Map format and SAMtools. Bioinformatics. 2009;25: 2078-2079.

45. Stanke M, Diekhans M, Baertsch R, Haussler D. Using native and syntenically mapped cDNA alignments to improve de novo gene finding. Bioinformatics. 2008;24: 637-644.

46. Liao Y, Smyth GK, Shi W. The R package Rsubread is easier, faster, cheaper and better for alignment and quantification of RNA sequencing reads. Nucleic Acids Res. 2019;47: e47.

47. Chen Y, Lun ATL, Smyth GK. From reads to genes to pathways: differential expression analysis of RNA-Seq experiments using Rsubread and the edgeR quasi-likelihood pipeline. F1000Res. 2016;5: 1438. 
48. Anders S, Huber W. Differential expression analysis for sequence count data. Genome Biol. 2010;11: R106.

49. Bullard JH, Purdom E, Hansen KD, Dudoit S. Evaluation of statistical methods for normalization and differential expression in mRNA-Seq experiments. BMC Bioinformatics. 2010;11: 94.

50. Robinson MD, Oshlack A. A scaling normalization method for differential expression analysis of RNA-seq data. Genome Biol. 2010;11: R25.

51. Law CW, Chen Y, Shi W, Smyth GK. voom: Precision weights unlock linear model analysis tools for RNA-seq read counts. Genome Biol. 2014;15: R29.

52. Phipson B, Lee S, Majewski IJ, Alexander WS, Smyth GK. ROBUST HYPERPARAMETER ESTIMATION PROTECTS AGAINST HYPERVARIABLE GENES AND IMPROVES POWER TO DETECT DIFFERENTIAL EXPRESSION. Ann Appl Stat. 2016;10: 946-963.

53. Ritchie ME, Phipson B, Wu D, Hu Y, Law CW, Shi W, et al. limma powers differential expression analyses for RNA-sequencing and microarray studies. Nucleic Acids Res. 2015;43: e47.

54. Mi H, Muruganujan A, Ebert D, Huang X, Thomas PD. PANTHER version 14: more genomes, a new PANTHER GO-slim and improvements in enrichment analysis tools. Nucleic Acids Res. 2019;47: D419-D426.

55. Mallard F, Jakšić AM, Schlötterer C. Contesting the evidence for non-adaptive plasticity. Nature. 2018;555: E21-E22.

56. Sujeeth N, Mehterov N, Gupta S, Qureshi MK, Fischer A, Proost S, et al. A novel seed plants gene regulates oxidative stress tolerance in Arabidopsis thaliana. Cell Mol Life Sci. 2020;77: 705-718.

57. Zhang S, Liu Y, Yu B. PRL1, an RNA-binding protein, positively regulates the accumulation of miRNAs and siRNAs in Arabidopsis. PLoS Genet. 2014;10: e1004841.

58. Shiu SH, Bleecker AB. Receptor-like kinases from Arabidopsis form a monophyletic gene family related to animal receptor kinases. Proc Natl Acad Sci U S A. 2001;98: 10763-10768.

59. Van der Does D, Boutrot F, Engelsdorf T, Rhodes J, McKenna JF, Vernhettes S, et al. The Arabidopsis leucine-rich repeat receptor kinase MIK2/LRR-KISS connects cell wall integrity sensing, root growth and response to abiotic and biotic stresses. PLoS Genet. 2017;13: e1006832.

60. Zhao C, Zayed O, Yu Z, Jiang W, Zhu P, Hsu C-C, et al. Leucine-rich repeat extensin proteins regulate plant salt tolerance in Arabidopsis. Proc Natl Acad Sci U S A. 2018;115: 13123-13128.

61. Belkhadir Y, Yang L, Hetzel J, Dangl JL, Chory J. The growth-defense pivot: crisis management in plants mediated by LRR-RK surface receptors. Trends Biochem Sci. 2014;39: 447-456.

62. Sultan SE, Spencer HG. Metapopulation structure favors plasticity over local adaptation. Am Nat. 2002;160: 271-283.

63. Scheiner SM. Genetics and evolution of phenotypic plasticity. Annu Rev Ecol Syst. 1993;24: 35-68.

64. Baucom RS, Mauricio R. Fitness costs and benefits of novel herbicide tolerance in a noxious weed. 
Proceedings of the National Academy of Sciences. 2004;101: 13386-13390.

65. Baucom RS, Mauricio R. Defence against the herbicide RoundUp ${ }^{\circledR}$ predates its widespread use. Evol Ecol Res. 2010;12: 131-141.

66. Kawecki TJ, Lenski RE, Ebert D, Hollis B, Olivieri I, Whitlock MC. Experimental evolution. Trends Ecol Evol. 2012;27: 547-560.

67. Trapnell C. Defining cell types and states with single-cell genomics. Genome Res. 2015;25: 1491-1498.

68. van Gestel J, Weissing FJ. Is plasticity caused by single genes? Nature. 2018;555: E19-E20.

69. Duhoux A, Carrère S, Gouzy J, Bonin L, Délye C. RNA-Seq analysis of rye-grass transcriptomic response to an herbicide inhibiting acetolactate-synthase identifies transcripts linked to non-target-site-based resistance. Plant Mol Biol. 2015;87: 473-487.

70. Serra A-A, Couée I, Heijnen D, Michon-Coudouel S, Sulmon C, Gouesbet G. Genome-Wide Transcriptional Profiling and Metabolic Analysis Uncover Multiple Molecular Responses of the Grass Species Lolium perenne Under Low-Intensity Xenobiotic Stress. Front Plant Sci. 2015;6: 1124.

71. Wright AA, Sasidharan R, Koski L, Rodriguez-Carres M, Peterson DG, Nandula VK, et al. Transcriptomic changes in Echinochloa colona in response to treatment with the herbicide imazamox. Planta. 2018;247: 369-379.

72. Zhao N, Li W, Bai S, Guo W, Yuan G, Wang F, et al. Transcriptome Profiling to Identify Genes Involved in Mesosulfuron-Methyl Resistance in Alopecurus aequalis. Front Plant Sci. 2017;8: 1391.

73. An J, Shen X, Ma Q, Yang C, Liu S, Chen Y. Transcriptome profiling to discover putative genes associated with paraquat resistance in goosegrass (Eleusine indica L.). PLoS One. 2014;9: e99940.

74. Leslie T, Baucom RS. De novo assembly and annotation of the transcriptome of the agricultural weed Ipomoea purpurea uncovers gene expression changes associated with herbicide resistance. G3 . 2014;4: 2035-2047.

75. Kohlhase DR, O'Rourke JA, Owen MDK, Graham MA. Using RNA-seq to characterize responses to 4-hydroxyphenylpyruvate dioxygenase (HPPD) inhibitor herbicide resistance in waterhemp (Amaranthus tuberculatus). BMC Plant Biol. 2019;19: 182. 